\title{
Efeito do óleo de capim limão (Cymbopogon flexuosus Stapf) no controle do carrapato dos bovinos
}

AGNOLIN, C.A..$^{1 *}$; OLIVO, C.J. ${ }^{1}$; PARRA, C. L. C. ${ }^{1}$

${ }^{1}$ Universidade Federal de Santa Maria (UFSM), Centro de Ciências Rurais, Departamento de Zootecnia, Santa Maria-RS, Brasil, 97105-900, "caiozoot2002@yahoo.com.br

RESUMO: Esta pesquisa foi conduzida com o objetivo de avaliar o efeito in vitro e in vivo do óleo essencial de capim limão (Cymbopogon flexuosus Stapf) sobre o carrapato dos bovinos [Rhipicephalus (Boophilus) microplus]. Na experimentação in vitro foi utilizado o grupo controle negativo e oito concentrações do óleo de capim-limão $(0,5 ; 1 ; 2 ; 5 ; 10 ; 20 ; 50 ; 100 \%)$, em fêmeas ingurgitadas do carrapato. A eficácia de controle foi de $0 ; 20 ; 39 ; 99,5 ; 100 ; 100 ; 100$; 100 e 100\%, respectivamente. Para a experimentação in vivo foram constituídos três grupos (controle negativo, óleo de capim limão a 2,0\% - nível estimado mediante análise de regressão, correspondendo a $95 \%$ de eficácia de controle do carrapato da pesquisa in vitro e amitraz a $0,025 \%$ ), com dezoito vacas da raça Holandesa. Antes (média dos dias $-3,-2,-1$ ) e após a aplicação do produto $(1,2,3,5,7,10,14$ e 21 dias), foram contadas fêmeas ingurgitadas do carrapato. A eficácia de controle foi de 0; 54 e 74,5\%, respectivamente, 21dias após o tratamento. Após a aplicação dos tratamentos (controle negativo $x$ tratamento fitoterápico), foram avaliadas as variáveis fisiológicas: frequência cardíaca, respiratória, temperatura do globo ocular e temperatura da pele; os resultados foram similares entre os tratamentos.

Palavras-chave: Acaricida, fitoterápico, vacas leiteiras, variáveis fisiológicas.

\begin{abstract}
Effect of lemongrass (Cymbopogon flexuosus Stapf) oil on the control of cattle ticks. This research was aimed at evaluating in vitro and in vivo effects of lemongrass (Cymbopogon flexuosus Stapf) oil on cattle ticks (Rhipicephalus (Boophilus) microplus). Negative control group and eight concentrations of lemongrass oil $(0.5 ; 1 ; 2 ; 5 ; 10 ; 20 ; 50 ; 100 \%)$, were used on in vitro trials with engorged female ticks. The efficacy of control ticks was $0 ; 20 ; 39$; 99.5; 100; 100; 100; 100 and $100 \%$, respectively. For the in vivo trial, eighteen Holstein cows were allocated to three groups (negative control, lemongrass oil at $2.0 \%$ - level estimated by regression analysis, accounting for $95 \%$ efficacy of control ticks on in vitro trial, and amitraz at $0.025 \%$ ). Engorged female ticks were counted before (mean of days $-3,-2,-1$ ) and after treatment $(1,2,3,5,7,10,14$ and 21 days). The efficacy of control ticks was $0 ; 54$ and $74.5 \%$, respectively, at 21 days after treatment. Physiologic variables were evaluated after treatments (negative control $x$ phytotherapic treatment). Similar results were found between the treatments for physiological variables.
\end{abstract}

Keywords:Acaricide, lactating cows, physiological variables, phytotherapy.

\section{INTRODUÇÃO}

O carrapato Rhipicephalus (Boophilus) microplus é o mais importante ectoparasito em áreas de exploração pecuária, tanto em regiões tropicais quanto subtropicais, sendo responsável por severas perdas econômicas (ATHAYDE et al., 2001). No Brasil, o carrapato esta presente em praticamente todos os rebanhos destinados à produção leiteira (LEITE \& ROCHA, 1999). Essa espécie, além de causar espoliação sanguínea em virtude do hematofagismo, lesa a pele, diminui a produção de leite e é o principal transmissor de agentes patogênicos para os bovinos (BITTENCOURT et al., 1999; JONSSON et al., 2001).

O uso de acaricidas é o principal meio de controle do carrapato (VARGAS et al., 2003), porém, o seu emprego nem sempre é feito de maneira correta (FURLONG et al., 2004), tornando os carrapatos resistentes aos produtos carrapaticidas (CAMILLO 
et al., 2009), além de possíveis problemas de saúde pública, devido à não observância dos períodos de carência dos produtos utilizados, (HEIMERDINGER, et al., 2006; MENDES, et al., 2007), especialmente em rebanhos de bovinos leiteiros (OLIVEIRA \& AZEVEDO, 2002).

Como técnicas alternativas não-químicas, incluem-se a criação de animais geneticamente mais resistentes, o desenvolvimento de vacinas, o manejo da pastagem com alternância de espécies, o controle biológico (LEAL at al., 2003) e também a utilização de extratos vegetais (CHAGAS, 2004), considerando que os produtos fitoterápicos apresentam baixo custo e são de fácil disponibilidade na maioria das regiões (AVANCINI, 1994; CHUNGSAMARNYART \& JIWAJINDA, 1992; HEIMERDINGER et al., 2006).

Dentre as espécies vegetais recomendadas destaca-se o capim limão, sendo que a mais difundida é a Cymbopogon citratus Stapf., enquanto a Cymbopogon flexuosus Stapf, apesar de ser menos cultivada, apresenta maior produção de biomassa e elevado teor de citral (CASTRO \& RAMOS, 2002). As pesquisas conduzidas com essas plantas, além de serem escassas, apresentam variação na forma de obtenção das soluções, que pode ser por extração aquosa e alcoólica, usando-se a planta inteira (HEIMERDINGER et al., 2006) ou somente a parte aérea (BROGLIO-MICHELETTI et al., 2009). Também há poucos estudos quanto ao uso dos componentes estruturais da planta e dos óleos essenciais. Os resultados das pesquisas apontam normalmente para efeito sobre o controle de ácaros, embora com elevada variabilidade de resultados.

Assim, o objetivo do trabalho foi avaliar a eficácia do óleo essencial do capim limão ( $C$. flexuosus), em experimentações in vitro e in vivo, em bovinos leiteiros como carrapaticida e verificar possíveis efeitos do produto em variáveis fisiológicas dos animais.

\section{MATERIAL E MÉTODOS}

O capim limão utilizado (Cymbopogon flexuosus Stapf) foi cultivado na mesorregião Noroeste do Rio Grande do Sul. O óleo foi extraído da parte aérea de plantas frescas na usina de extração de óleos essenciais por arraste a vapor no Pólo Oleoquímico de Três Passos, pertencente a Universidade do Noroeste do Estado do Rio Grande do Sul (UNIJUÍ), tendo apresentado rendimento de $1,2 \%$, aproximadamente. A análise cromatográfica do óleo foi realizada no Departamento de Química da Universidade Federal de Santa Maria (UFSM), apresentando como princípio ativo mais importante o citral com $90,6 \%$ (geranial $51,7 \%$ e neral $38,9 \%$ ). Outros compostos perfizeram $9,4 \%$.

Para a experimentação in vitro foram coletadas, em dezembro de 2009, fêmeas ingurgitadas, retiradas de animais da raça Holandesa, naturalmente infestados, pertencentes ao Laboratório de Bovinocultura de Leite do Departamento de Zootecnia da Universidade Federal de Santa Maria, sendo que o último tratamento das vacas contra o carrapato foi realizado em maio de 2009.

Os tratamentos foram constituídos pelo grupo controle e por diferentes concentrações do óleo de capim limão $(0,5 ; 1,0 ; 2,0 ; 5,0 ; 10,0 ; 20,0$; 50,0 e $100,0 \%$ ). Para o grupo controle (testemunha, sem óleo) e para a complementação das demais soluções (até o nível de $50 \%$ ) usou-se água destilada. As fêmeas ingurgitadas foram colocadas aleatoriamente em grupos de 10 em placas de Petri, sendo usadas três placas/tratamento, submetidas ao Teste de imersão de fêmeas ingurgitadas (DRUMMOND et al., 1973), realizado no Laboratório de Doenças Parasitárias da UFSM. O delineamento experimental utilizado foi o inteiramente casualizado, com três repetições (placas de Petri).

$O$ experimento in vivo foi realizado no Laboratório de Bovinocultura de Leite (DZ, UFSM). Foram constituídos três tratamentos: amitraz, a 0,025\% (grupo controle positivo), óleo de capim limão a 2,0\% e testemunha (grupo controle negativo). A opção pelo Amitraz deveu-se à eficácia in vitro de $98,2 \%$ no controle da cepa utilizada no presente experimento. Para a concentração com óleo de capim limão, o nível utilizado de 2,0\%, teve como base os resultados da experimentação in vitro, sendo submetidos à análise de regressão polinomial, estimando-se um valor de $95 \%$ de eficácia. O óleo de capim limão utilizado foi o mesmo daquele utilizado na experimentação in vitro.

No experimento foram utilizadas 18 vacas em lactação da raça Holandesa, com cerca de $530 \mathrm{~kg}$ de peso vivo e produção média de 21 $\mathrm{kg}$ de leite/vaca/dia, manejadas em conjunto em pastagens perenes de ciclo estival. Os animais foram submetidos à ordenha mecânica, duas vezes ao dia. A base da alimentação foi constituída de pastagens perenes de ciclo estival. A complementação alimentar foi feita com concentrado $(18 \%$ PB) à razão de $5 \mathrm{~kg} / \mathrm{vaca} / \mathrm{dia}$, dividida entre as ordenhas da manhã e da tarde. Foram utilizadas seis vacas por tratamento. O critério para utilização de cada animal foi a infestação de carrapatos, sendo usadas vacas que apresentavam no mínimo dez teleóginas (média de três dias consecutivos). Os animais foram submetidos aos tratamentos em janeiro de 2010 , sendo que o último controle do carrapato foi feito em maio de 2009. O preparo das soluções foi feito momentos antes e aplicadas, após a ordenha da tarde, utilizando pulverizador costal. Para o preparo da solução com o fitoterápico fez-se uma pré mistura

Rev. Bras. PI. Med., Campinas, v.16, n.1, p.77-82, 2014. 
do óleo com água. Para ambos os tratamentos, a quantidade de calda usada foi de 4 litros/vaca.

Nas avaliações foram efetuadas contagens de carrapato, considerando-se os instares com tamanho superior a $4,5 \mathrm{~mm}$ de comprimento, na metade do corpo do animal, multiplicando-se o valor por dois para a obtenção da infestação total (WHARTON et al., 1970). As contagens foram feitas no $1^{\circ}, 2^{\circ}, 3^{\circ}, 5^{\circ}, 7^{\circ}, 10^{\circ}, 14^{\circ}$ e $21^{\circ}$ dia após a aplicação dos produtos. Para calcular a eficácia do produto foi utilizada a seguinte fórmula: Eficácia $=[(№$ de teleóginas de pré-tratamento - № de teleóginas do dia de pós-tratamento) ${ }^{*} 100$ / № de teleóginas de pré-tratamento]. $\mathrm{O}$ delineamento experimental utilizado foi o inteiramente casualizado, sendo que, para a variável "número de teleóginas", os dados foram analisados após a transformação logarítmica de base 10 .

Para a análise das variáveis fisiológicas, conduziu-se outra experimentação, mantendo-se os mesmos tratamentos, sendo aferidas antes e às três, seis e 24 horas após a aplicação dos tratamentos. As frequências cardíaca e respiratória foram verificadas mediante auscultação com estetoscópio. Para aferir as temperaturas de globo ocular e da superfície da pele utilizou-se equipamento com infravermelho ( $\mathrm{TI}$ 890 Instron com resolução de 50x). A opção de uso desse equipamento deve-se a sua confiabilidade e por ser um procedimento não invasivo (KOTRBA et al., 2007).

O delineamento experimental foi o inteiramente casualizado, sendo os tratamentos constituídos pelos grupos controle negativo e positivo (com produto químico a base de amitraz) além da solução com $2 \%$ de óleo de capim limão, estimada a partir da análise de regressão, para controlar $95 \%$ do carrapato. As unidades experimentais foram constituídas pelas vacas em lactação

Para ambos os experimentos (in vitro e in vivo) os dados foram submetidos à análise de variância e as médias comparadas pelo teste de Tukey ou $\mathrm{F}$ ao nível de $5 \%$ de probabilidade do erro. Foi utilizado o seguinte modelo matemático: $Y_{i j}=m+T_{i}+E_{i j}$, em que, $Y_{i j}$ representa a variável dependente; $\mathrm{i}=\mathrm{o}$ índice de tratamentos; $\mathrm{j}$ = $\mathrm{o}$ índice de repetições; $m$ é a média de todas as observações; $T_{i}$ corresponde ao efeito dos tratamentos; $E_{i j}$ é o erro experimental. Os dados foram analisados com auxílio do programa estatístico SAS (1997).

\section{RESULTADOS E DISCUSSÃO}

Com relação à experimentação in vitro (Tabela 1), observa-se que houve efeito $(P<0,05)$ dos níveis, a partir de $0,5 \%$ de óleo de capim limão, sobre a eclodibilidade dos ovos. Não houve eclodibilidade com níveis de concentração a partir de $5 \%$. Os dados submetidos à análise de regressão demonstram efeito quadrático com início descendente $y=134,564-91,129 x+12,837 x^{2} ; R^{2}=$ 0,$99 ; \mathrm{P}=0,0014$.

Para eficácia, houve efeito $(P<0,05)$ a partir da solução constituída com menor nível de óleo $(0,5 \%)$. A partir de $5 \%$ de óleo, a eficácia foi de $100 \%$. Os dados submetidos à análise de regressão demonstram efeito quadrático com início ascendente $y=-23,659+82,907 x-11,622 x^{2} ; R^{2}=$ 0,$97 ; P=0,0238$. Os resultados apontam que mesmo as baixas concentrações de óleo interferem no desenvolvimento do carrapato. Broglio-Micheletti et al. (2009), avaliando o extrato etanólico de folhas secas de capim santo (C. citratus) a $2 \%$, verificaram redução da eclodibilidade em $25,5 \%$ e eficácia de $18,35 \%$ no controle do carrapato.

Outros trabalhos mostram a mesma tendência como Silva et al. (2007), no qual o capim santo teve eficácia de $20,32 \%$, a partir do extrato puro de folhas frescas. Porém, a ação foi maior quando utilizado o extrato diluído com $50 \%$ de água e outro diluído com $50 \%$ de álcool, verificando controle de 35,7 e $47 \%$, respectivamente. Neste trabalho os autores apontam para um possível efeito potencializador do álcool, encontrando respaldo no trabalho feito por Chagas et al. (2002), quando

TABELA 1. Médias porcentuais de eclodibilidade das larvas e da eficácia das soluções constituídas pelo controle e por diferentes concentrações de óleo de capim limão (Cymbopogon flexuosus Stapf) aplicado sobre teleóginas de Rhipicephalus (Boophilus) microplus pelo Teste de imersão de teleóginas. Santa Maria, RS, 2011.

\begin{tabular}{ccc}
\hline $\begin{array}{c}\text { Óleo de capim } \\
\text { limão (\%) }\end{array}$ & $\begin{array}{c}\text { Eclodibilidade } \\
(\%)\end{array}$ & $\begin{array}{c}\text { Eficácia do } \\
\text { produto (\%) }\end{array}$ \\
\hline 0 (controle) & $99,0^{\mathrm{a}}$ & $0,0^{\mathrm{d}}$ \\
0,5 & $90,0^{\mathrm{b}}$ & $20,0^{\mathrm{c}}$ \\
1,0 & $60,0^{\mathrm{c}}$ & $39,0^{\mathrm{b}}$ \\
2,0 & $2,0^{\mathrm{d}}$ & $99,5^{\mathrm{a}}$ \\
5,0 & $0,0^{\mathrm{d}}$ & $100,0^{\mathrm{a}}$ \\
10,0 & $0,0^{\mathrm{d}}$ & $100,0^{\mathrm{a}}$ \\
20,0 & $0,0^{\mathrm{d}}$ & $100,0^{\mathrm{a}}$ \\
50,0 & $0,0^{\mathrm{d}}$ & $100,0^{\mathrm{a}}$ \\
100,0 & $0,0^{\mathrm{d}}$ & $100,0^{\mathrm{a}}$ \\
& & \\
CV (\%) & 4,47 & 1,47 \\
\hline Médias com letras distintas, na coluna, indicam diferença \\
significativa (P<0,05) pelo teste de tukey; Óleo de capim limão \\
oriundo do Laboratório de Óleos Essenciais (UNIJUÍ - ljú, RS), \\
do Pólo Oleoquímico de Três Passos, RS. CV= Coeficiente de \\
variação.
\end{tabular}


comparou diferentes tipos de solventes. Também Heimerdinger et al. (2009) verificaram eficácia de controle de teleóginas, usando macerado da planta inteira, com extração alcoólica, correspondendo a 23,08 e $37,5 \%$ de matéria natural, com controle de 87,6 e $92,6 \%$, respectivamente.

A ação acaricida deve-se, provavelmente, ao princípio ativo citral (mistura isomerica de neral e geranial) que é o principal constituinte do óleo do capim limão (COSTA et al., 2005). A mortalidade dos ácaros depende da concentração do óleo essencial utilizado, sendo que, quanto maior a concentração melhor será esta ação acaricida (MEZA \& TABORDA, 2010).

Para eficácia no controle de teleóginas na experimentação in vivo (Tabela 2), observase que o produto a base de amitraz, apresentou eficácia de $100 \%$ no 3o dia pós-banho, havendo, no entanto, presença de teleóginas a partir do 5 dia. Para o grupo tratado com o fitoterápico verificou-se que houve controle parcial, havendo, inicialmente, efeito mais lento se comparado com o

TABELA 2. Efeito da solução aquosa contendo óleo de capim limão (Cymbopogon flexuosus Stapf) e do amitraz no controle do carrapato Rhipicephalus (Boophilus) microplus em bovinos da raça Holandesa. Santa Maria, RS, 2011.

\begin{tabular}{|c|c|c|c|c|}
\hline \multirow{2}{*}{ Avaliações } & \multicolumn{3}{|c|}{ Número de teleóginas/vaca ${ }^{1}$} & \multirow{2}{*}{ CV (\%) } \\
\hline & Controle $^{2}$ & Capim limão ${ }^{3}$ & Amitraz $^{4}$ & \\
\hline Pré-tratamento & 22,5 & 18,0 & 29,0 & 8,0 \\
\hline \multicolumn{5}{|l|}{ Pós-tratamento } \\
\hline 1o dia & $25,8^{\mathrm{a}}$ & $21,2^{\mathrm{ab}}$ & $11,0^{\mathrm{b}}$ & 14,5 \\
\hline $2^{\circ} \mathrm{dia}$ & $29,2^{\mathrm{a}}$ & $13,7^{\mathrm{b}}$ & $2,0^{c}$ & 14,4 \\
\hline 3ㅇ dia & $32,6^{a}$ & $12,5^{\mathrm{b}}$ & $0,0^{c}$ & 15,7 \\
\hline 5o dia & $29,0^{a}$ & $7,2^{\mathrm{b}}$ & $1,0^{c}$ & 19,1 \\
\hline 7ㅇdia & $30,6^{a}$ & $7,2^{b}$ & $1,2^{\mathrm{c}}$ & 14,9 \\
\hline $10^{\circ} \mathrm{dia}$ & $29,2^{a}$ & $7,7^{\mathrm{b}}$ & $1,7^{\circ}$ & 14,5 \\
\hline $14^{\circ}$ dia & $32,2^{a}$ & $6,2^{\mathrm{b}}$ & $0,7^{c}$ & 17,9 \\
\hline $21^{\circ} \mathrm{dia}$ & $34,4^{\mathrm{a}}$ & $8,2^{b}$ & $7,0^{\mathrm{b}}$ & 13,9 \\
\hline Média Pós-tratamento & $30,2^{\mathrm{a}}$ & $10,5^{\mathrm{b}}$ & $3,0^{c}$ & 24,4 \\
\hline \multicolumn{5}{|c|}{ Eficácia no controle de teleóginas (\%) } \\
\hline Pré-tratamento & 0,0 & 0,0 & 0,0 & 0,0 \\
\hline \multicolumn{5}{|l|}{ Pós-tratamento } \\
\hline 10 dia & $0,0^{\mathrm{b}}$ & $0,0^{\mathrm{b}}$ & $63,0^{\mathrm{a}}$ & 31,8 \\
\hline $2^{\circ}$ dia & $0,0^{c}$ & $24,5^{b}$ & $93,2^{\mathrm{a}}$ & 18,4 \\
\hline 3ㅇ dia & $0,0^{c}$ & $29,7^{b}$ & $100,0^{a}$ & 15,6 \\
\hline 5o dia & $0,0^{c}$ & $60,0^{b}$ & $96,7^{\mathrm{a}}$ & 12,1 \\
\hline 7ㅇ dia & $0,0^{c}$ & $60,0^{\mathrm{b}}$ & $96,0^{a}$ & 11,9 \\
\hline $10^{\circ} \mathrm{dia}$ & $0,0^{c}$ & $57,0^{\mathrm{b}}$ & $93,7^{\mathrm{a}}$ & 9,1 \\
\hline $14^{\circ} \mathrm{dia}$ & $0,0^{c}$ & $66,2^{b}$ & $97,0^{\mathrm{a}}$ & 20,2 \\
\hline $21^{\circ} \mathrm{dia}$ & $0,0^{c}$ & $54,0^{\mathrm{b}}$ & $74,5^{\mathrm{a}}$ & 20,6 \\
\hline Média Pós-tratamento & $0,0^{c}$ & $43,9^{b}$ & $89,2^{\mathrm{a}}$ & 37,6 \\
\hline
\end{tabular}

Médias com letras distintas, na linha, indicam diferença significativa $(P<0,05)$ pelo teste de Tukey; ${ }^{1}$ dados analisados após a transformação logarítmica de base 10; ${ }^{2}$ Tratamento constituído pelo controle = grupo controle negativo; ${ }^{3}$ Capim limão $=$ solução aquosa contendo $2 \%$ de óleo de capim limão; ${ }^{4}$ Amitraz $=$ a $0,025 \%$ de amitraz, grupo controle positivo. 
produto químico. A melhor eficácia, próximo a $66 \%$, no 14 을 dia pós-banho, é inferior à avaliação feita in vitro no qual o controle foi de $95 \%$ para a mesma concentração utilizada ( $2 \%$ de óleo). Os resultados foram superiores aos encontrados por Heimerdinger et al. (2006), ao utilizarem solução feita com extrato alcoólico, correspondendo a $2,72 \%$ de planta de capim limão, que verificaram controle de 40,3; 46,5 e $41,4 \%$ no $3^{\circ}, 7^{\circ}$ e $14^{\circ}$ dia pós-banho.

Os resultados obtidos in vivo podem ser atribuídos à volatilidade do óleo essencial. Em trabalho em que foi avaliada a repelência e a toxicidade de oito constituintes de óleos essenciais, o citral demonstrou boa ação numa concentração de 10 a $50 \mu \mathrm{g} / \mathrm{cm}^{2}$ durante 3 dias, porém, a ação durou apenas um curto período de tempo em virtude da sua volatilidade (ZHU et al., 2001).

Assim, é possível que na avaliação in vivo possa haver melhor condição de volatilidade, devido à aplicação do produto sob aspersão, implicando na formação de pequenas gotículas facilitando a volatilização devido ao aumento da superfície específica.

Para as variáveis fisiológicas (Tabela 3), não foram observadas diferenças entre o controle e os animais banhados com a solução constituída por óleo de capim limão. Os valores na avaliação inicial, antecedendo os banhos (às 9:00h), demonstraram que os animais apresentavam variáveis fisiológicas próximas da normalidade (SMITH, 1994). Observouse, no entanto, que nos dois grupos houve elevação dos valores das frequências cardíaca e respiratória, com pico na avaliação feita às 15:00h; também houve elevação das temperaturas do globo ocular e da pele entre às 12:00 e às 15:00h do dia. Esses resultados estão associados à elevação da temperatura do ar, apontando que os animais apresentavam estresse térmico. Segundo Silva (2002) em temperatura superior a $24^{\circ} \mathrm{C}$, verifica-se desconforto térmico, influenciando no metabolismo, com aumento das frequências cardíaca e respiratória, e diminuição do consumo.

\section{CONCLUSÕES}

As soluções contendo níveis crescentes de óleo de capim limão avaliados in vitro apresentam efeito quadrático ascendente no controle do carrapato.

A solução contendo $2,0 \%$ de óleo de capim limão apresenta controle parcial do carrapato dos bovinos, apresentando baixa associação com os níveis avaliados in vitro.

Há similaridade para as variáveis fisiológicas

TABELA 3. Variáveis fisiológicas de vacas da raça Holandesa, submetidas à aplicação de solução aquosa contendo 2,0\% de óleo do capim limão (Cymbopogon flexuosus Stapf) para o controle do carrapato Rhipicephalus (Boophilus) microplus. Santa Maria, RS, 2011.

\begin{tabular}{|c|c|c|c|c|c|}
\hline \multirow[b]{2}{*}{$\begin{array}{c}\text { Período } \\
\text { (hora do dia) }\end{array}$} & \multirow[b]{2}{*}{ Grupo } & \multicolumn{4}{|c|}{ Variáveis fisiológicas } \\
\hline & & $\begin{array}{c}\text { Frequência } \\
\text { cardíaca (bat/ } \\
\text { min.) }\end{array}$ & $\begin{array}{l}\text { Frequência } \\
\text { respiratória } \\
\text { (mov/min.) }\end{array}$ & $\begin{array}{c}\text { Temperatura } \\
\text { globo ocular } \\
\left({ }^{\circ} \mathrm{C}\right)\end{array}$ & $\begin{array}{c}\text { Temperatura da } \\
\text { pele } \\
\left({ }^{\circ} \mathrm{C}\right)\end{array}$ \\
\hline \multirow{2}{*}{ Antes (9:00) } & Controle $^{1}$ & 70,0 & 33,3 & 30,3 & 27,0 \\
\hline & Capim limão² & 73,3 & 33,3 & 31,0 & 25,0 \\
\hline \multirow{2}{*}{3 horas $(12: 00)$} & Controle & 73,0 & 49,3 & 33,9 & 31,0 \\
\hline & Capim limão & 77,3 & 40,0 & 33,6 & 32,0 \\
\hline \multirow{2}{*}{6 horas $(15: 00)$} & Controle & 77,0 & 54,0 & 30,0 & 27,6 \\
\hline & Capim limão & 70,6 & 46,0 & 31,0 & 26,3 \\
\hline \multirow{2}{*}{24 horas $(9: 00)$} & Controle & 65,0 & 27,6 & 28,6 & 24,0 \\
\hline & Capim limão & 64,6 & 26,6 & 28,0 & 21,3 \\
\hline \multirow{2}{*}{ Média } & Controle & 71,2 & 41,0 & 30,7 & 27,4 \\
\hline & Capim limão & 71,5 & 34,0 & 30,9 & 26,1 \\
\hline $\mathrm{CV}(\%)$ & & 12,7 & 37,6 & 6,7 & 14,0 \\
\hline
\end{tabular}

Diferença não significativa entre os grupos $(\mathrm{P}>0,05)$ pelo teste $\mathrm{F} ;{ }^{1}$ controle = grupo controle negativo; ${ }^{2}$ capim limão = solução aquosa contendo $2 \%$ de óleo de capim limão; Períodos: antes da aplicação, às 3 e 6 horas correspondem ao dia 27/06/2010 e 24 horas corresponde ao dia 28/06/2010. Temperatura mínima e máxima de 16 e $28^{\circ} \mathrm{C}$ e de 14 e $21^{\circ} \mathrm{C}$, respectivamente para o dia 27 e 28/06/2010. 
entre os grupos de animais não tratados e os que foram banhados com solução contendo óleo de capim limão.

\section{REFERÊNCIA}

ATHAYDE, A.C.R. et al. Fungos entomopatogênicos: uma alternativa para o controle do carrapato bovino: Boophilus microplus. Biotecnologia Ciência e Desenvolvimento, v.21, n.1, p.12-15, 2001.

AVANCINI, C.A.M. Sanidade animal na agroecologia: atitudes ecológicas de sanidade animal e plantas medicinais em Medicina Veterinária. Porto Alegre: Fundação Gaia, 1994. 46p.

BITTENCOURT, V.R.E.P. et al. Mecanismo de infecção do fungo Metarhizium anisopliae no carrapato Boophilus microplus em condições experimentais. Ciência Rural, v.29, n.2, p.351-354, 1999.

BROGLIO-MICHELETTI, S.M.F. et al. Extratos de plantas no controle de Rhipicephalus (Boophilus) microplus (Canestrini, 1887) (Acari: Ixodidae) em laboratório. Revista Brasileira de Parasitologia Veterinária, v.18, n.4, p.44-48, 2009.

CAMILLO, G. et al. Eficiência in vitro de acaricidas sobre carrapatos de bovinos no Estado do Rio Grande do Sul, Brasil. Ciência Rural, v.39, n.2, p.490-495, 2009.

CASTRO, L. O. de ; RAMOS, R. L. D. Principais gramíneas produtoras de óleos essenciais. Porto Alegre: FEPAGRO, 2002. 31 p. (Boletim FEPAGRO, 11).

CHAGAS, A.C.S. et al. Efeito acaricida de óleos essenciais e concentrados emulsionáveis de Eucalyptus spp em Boophilus microplus. Brazilian Journal of Veterinary Research and Animal Science, v.39, n.5, p.247-253, 2002.

CHAGAS, A.J.S. Controle de parasitas utilizando extratos vegetais. Revista Brasileira de Parasitologia Veterinária, v.13, n.1, p.156-60, 2004.

CHUNGSAMARNYART, N.; JIWAJINDA, S. Acaricidal activity of volatile oil from lemon and citronella grasses on tropical cattle ticks. Kasetsart Journal (Natural Science), v.26, n.5, p.46-51, 1992.

COSTA, L.C.B. et al. Secagem e fragmentação da matéria seca no rendimento e composição do óleo essencial de capim limão. Horticultura Brasileira, v.23, n.4, p.956-959, 2005.

DRUMMOND, R.O. et al. Boophilus annulatus and Boophilus microplus: Laboratory test of insecticides. Journal Economical Entomology, v.66, n.1, p.130133, 1973.

FURLONG, J. et al. Controle estratégico do carrapato dos bovinos. A Hora Veterinária, v.23, n.137, p.53-56, 2004.

HEIMERDINGER, A. et al. Extrato alcoólico de capimcidreira no controle do Boophilus microplus em bovinos. Revista Brasileira de Parasitologia Veterinária, v.15, n.1, p.37-39, 2006.

HEIMERDINGER, A. et al. Extratos de capim-cidreira e amitraz em teste in vitro sobre o carrapato bovino.
Livestock Research for Rural Development, v.21, n.1, artigo 5, 2009.

JONSSON, N. N. et al. An estimate of the economic effects of cattle tick (Boophilus microplus) infestation on Queensland dairy farms. Australian Veterinary Journal, v.79, n.12, p.826-831, 2001.

KOTRBA, R. et al. Comparison between the coat temperature of the eland and dairy cattle by infrared thermography. Journal of Thermal Biology, v.32, p.355-359, 2007.

LEAL, A.T. et al. Perspectivas para o controle do carrapato bovino. Acta Scientiae Veterinariae, v.31, n.1, p.1-11, 2003.

LEITE, R.C.; ROCHA, C.M.D.M. Contagem de carrapatos em bovinos no momento do banho carrapaticida em rebanhos leiteiros do município de Divinólopis/ MG. Arquivo Brasileiro de Medicina Veterinária e Zootecnia, v.51, n.1, p.41-42, 1999.

MENDES, M.C.et al. Determinação da frequência de realização de bioensaios para o monitoramento da resistência do carrapato Boophilus microplus (Acari: Ixodidae). Arquivos do Instituto Biológico, v.74, n.2, p. 87-93, 2007.

MEZA, D.L.M.; TABORDA, M. Composición química y activida del aceite esencial de Cymbopongon citratus Stapf contra el acaro intradomiciliario Dermatophagoides farinae (Acari: Pyroglyphidae). Biosalud, v.9, n.2, p.2131, 2010.

OLIVEIRA, A.A; AZEVEDO, H.C. Resistência do carrapato Boophilus microplus a carrapaticidas em bovinos de leite na região dos tabuleiros costeiros de Sergipe. Revista Científica Rural, v.7, n.2, p.64-71, 2002.

SAS, Statistical Analysis System: User's Guide, Stat. 2.ed. Cary: SAS Institute, 1997. 456p.

SILVA, I. J. O. et al. Efeitos da climatização do curral de espera na produção de leite de vacas holandesas. Revista Brasileira de Zootecnia, v.31, n.5, p.20362042, 2002.

SILVA, W.W. et al. Efeitos do neem (Azadirachta indica A. Juss) e do capim santo [Cymbopogon citratus(DC) Stapf] sobre os parâmetros reprodutivos de fêmeas ingurgitadas de Boophilus microplus e Rhipicephalus sanguineus (Acari: Ixodidae) no semiárido paraibano. Revista Brasileira de Plantas Medicinais, v.9, n.3, p.1-5, 2007.

SMITH, B. P.Tratado de medicina interna de grandes animais. 2. ed. São Paulo: Manole, 1994. 1738 p.

VARGAS, M.S. et al. Avaliação in vitro de uma cepa de campo de Boophilus microplus (Acari: Ixodidae) resistente à Amitraz. Ciência Rural, v.33, n.4, p.737742, 2003

WHARTON, R.H. et al. Assessment of the efficiency of acaricides and their mode of aplication against the cattle tick Boophilus microplus. Australian Journal of Agricultural Research, v.21, n.5, p.985-1006, 1970.]

ZHU, B.C.R. et al. Evaluation of vetiver oil and seven insect-active essential oils against the formosan subterranean termite. Journal of Chemical Ecology, v.27, n.8, p.1617-1625, 2001. 